\title{
氧化石墨催化的酯化与酯交换反应
}

\author{
齐俊梅许跃龙马宁* 孙菲菲* \\ (天津大学理学院化学系 天津 300072)
}

\begin{abstract}
摘要 将氧化石墨用于催化羧酸与醇、酚的酯化反应以及醇与酯的酯交换反应，笁选了不同方法制得的 3 种氧化石墨， 发现改进 Hummers 法制备的氧化石墨对于羧酸与伯醇、仲醇的酯化以及醇与酯的酯交换反应有很高的催化活性，反应 条件温和, 产物分离提纯简便, 收率高; 其对羧酸与叔醇、酚的酯化反应催化活性较低. 氧化石墨作为一种固体弱酸性 催化剂, 制备简单, 价格低廉, 环境友好, 能与多种官能团兼容. 单独使用氧化石墨作催化剂, 能高效催化高级脂肪酸 的酯化及高级脂肪酸甘油酯的酯交换反应，这在生物柴油的制备领域具有潜在的应用价值.
\end{abstract}

关键词 氧化石墨; 催化剂; 酯化; 酯交换; 生物柴油

\section{Graphite Oxide-Catalyzed Esterification and Transesterification}

\author{
Qi, Junmei Xu, Yuelong $\quad$ Ma, Ning* Sun, Feifei* \\ (Department of Chemistry, School of Science, Tianjin University, Tianjin 300072)
}

\begin{abstract}
Graphite oxide (GO) was involved in the esterification of carboxylic acids with alcohols or phenols and the transesterification of esters with alcohols. Three types of GO prepared by different methods were screened and GO by modified Hummers method was found superior to the others. The esterification of primary and secondary alcohols afforded the corresponding esters in moderate to excellent yields under mild conditions. GO-catalyzed esterification of tertiary alcohols and phenols took place hardly. Moreover, GO showed highly catalytic activity for the transesterification. As a weakly acidic solid catalyst, GO is easily available, cheap, environmentally benign, and well tolerant to various functional groups. Only catalyzed by GO, the esterification of long-chain carboxylic acids and the transesterification of the glycerol ester of long-chain carboxylic acids underwent steadily. As such, GO-catalyzed esterification and transesterification could be used in the biodiesel preparation potentially.
\end{abstract}

Keywords graphite oxide; catalyst; esterification; transesterification; biodiesel

酯类化合物在医药、化工等领域有着广泛的应用 ${ }^{[1]}$, 酯化与酯交换反应一直是合成酯类化合物的主要途 径 ${ }^{[1]}$, 最近因其在制备生物柴油上的重要应用而被广泛 关注 ${ }^{[2]}$. 液体酸(如硫酸)是酯化反应常用催化剂, 但具 有对设备腐蚀性强, 难于从均相体系分离等缺点. 固体 酸等非均相催化剂具有反应选择性高, 易于从体系分 离, 产生废物少等优点, 近年来得到了广泛的应用, 如 高氟化离子交换树脂、杂多酸等 ${ }^{[3]}$ 是酯化反应较好的催 化剂, 但其价格较高且催化效果不如液体酸 ${ }^{[4]}$. 此外, 离子液体、脂肪酶等 ${ }^{[5]}$ 也常用作酯化反应的催化剂. 酯 交换反应通常由无机和有机碱催化. 最近硅胶负载金属 锆、氮杂环卡宾等 ${ }^{[6]}$ 也用作酯交换反应的催化剂, 一些 碳材料也被报道用于催化酯化反应和酯交换反应, 例如
高温处理的磺化糖类、磺化的石墨烯、硝酸氧化的活性 炭等 ${ }^{[7]}$.

近年来, 氧化石墨(GO)或氧化石墨烯(单层的氧化 石墨)作为一类新型材料得到国内外学者的广泛关注, 其本身可用于构建纳米材料, 还可经过还原制备石墨烯 (也称为还原的氧化石墨烯) 类纳米材料 ${ }^{[8]}$. 氧化石墨通 常是由石墨经强氧化剂氧化获得. 如传统 Hummers 法 ${ }^{[9]}$, 用 $\mathrm{KMnO}_{4}, \mathrm{NaNO}_{3}$ 和 $\mathrm{H}_{2} \mathrm{SO}_{4}$ 体系在 $98{ }^{\circ} \mathrm{C}$ 对石墨 进行氧化; 为提高氧化效果, 改进 Hummers 法在原来的 基础上加入过量的 $\mathrm{KMnO}_{4}$, 并在较低的温度下反应; Tour 法采用 $\mathrm{KMnO}_{4}, \mathrm{H}_{3} \mathrm{PO}_{4}$ 和 $\mathrm{H}_{2} \mathrm{SO}_{4}$ 作为氧化剂来制 备氧化石墨 ${ }^{[10]}$. 氧化石墨是一种无定形、非化学计量、 环境友好的固体, 具有吸湿性、弱酸性 ${ }^{[11]}$. 氧化石墨的

\footnotetext{
*E-mail: mntju@tju.edu.cn; sff@tju.edu.cn

Received December 25, 2012; revised February 28, 2013; published online April 3, 2013.

Project supported by the National Natural Science Foundation of China (No. 20802049).

国家自然科学基金(No. 20802049)资助项目.
} 
具体结构至今尚不明确, 仍存在一些争议, 但通常认为 氧化石墨的表面及边缘富含有羟基、羰基、羧基、环氧 基等含氧官能团, 一种被多数人接受的氧化石墨烯结构 模型如图 1 所示 ${ }^{[11]}$.

尽管氧化石墨在几十年前就已被制备, 但罕有单独 将其用于催化有机反应的报道, 直到 2010 年, Bielawski 等报道了氧化石墨催化醇的氧化以及炔的水合反应 ${ }^{[12]}$. 随后, 氧化石墨作为氧化反应催化剂被用于胺的氧化偶 联 ${ }^{[13]}$, 氧化石墨或氧化石墨烯作为固体弱酸催化剂成 功应用于催化吲哚与 $\alpha, \beta$-不饱和酮的 Friedel-Crafts 反 应、胺与 $\alpha, \beta$-不饱和化合物的 aza-Michael 加成、环氧衍 生物与醇的开环等有机反应 ${ }^{[14]}$. 我们课题组 ${ }^{[15]}$ 报道了 氧化石墨与分子篎结合用于饱和的芳环或杂芳环的脱 氢芳构化反应. 2011 年, 刘钢等 ${ }^{[16]}$ 报道了氧化石墨负载 的纳米 $\mathrm{CaO}$ 催化大豆油与甲醇的酯交换反应, 文中认 为作为活性 $\mathrm{CaO}$ 的受体材料, 氧化石墨可能起稳定 $\mathrm{CaO}$ 纳米粒子的作用, 但并未探讨氧化石墨本身的催化作 用.

鉴于氧化石墨单独作催化剂用于催化酯化以及酯 交换反应的系统研究未见报道, 本文将通过几种不同方 法制备的氧化石墨用于酯化反应, 比较篮选出催化活性 高者, 并改变酸和醇的结构进行催化反应, 发现催化剂 对底物中的多种官能团相容性好. 我们还将氧化石墨用 于酯交换反应, 发现其具有明显的催化效果. 由于单独 使用氧化石墨作催化剂, 不但对高级脂肪酸的酯化, 而 且对高级脂肪酸甘油酯的酯交换反应都有较高的催化 活性, 因此本研究结果在生物柴油的制备领域体现出潜 在的应用价值. 用于催化反应的氧化石墨经过 FT-IR, XRD, XPS 以及元素分析进行结构表征. 合成的酯类化 合物均为已知物, 经过 ${ }^{1} \mathrm{H}$ NMR 和 ${ }^{13} \mathrm{C}$ NMR 的分析表 征.

\section{1 结果与讨论}

\section{1 催化剂种类的优化选择}

我们以环己烷甲酸与甲醇的反应为模板，比较不同 类型的催化剂的催化效果(表 1). 表 1 的空白实验(表 1 , Entry 4)中不加催化剂, 转化率只有 $9 \%$. 以天然石墨(表 1, Entry 5)为催化剂, 转化率只有 6\%. 用传统 Hummers 法制备的氧化石墨(GO-1)以及用 Tour 法制备的氧化石 墨(GO-2)作为催化剂，转化率分别只有 $51 \%$ 和 $77 \%$. 用 改进 Hummers 法制备的氧化石墨(GO)作为催化剂, 转 化率高达 99\%，选择性接近 100\%. 因此，我们选用改进 Hummers 法制备的 GO 作为反应催化剂.

表 1 环己烷甲酸与甲醇的酯化反应 ${ }^{a}$

Table 1 Esterification of cyclohexanoic acid with methanol

\begin{tabular}{cccc}
\hline Entry & Catalyst & Conv. $^{b} / \%$ & Select. $^{c} / \%$ \\
\hline 1 & GO & 99 & $>99$ \\
2 & GO-1 $^{d}$ & 51 & 97 \\
3 & GO-2 $^{e}$ & 77 & 99 \\
4 & - & 9 & 97 \\
5 & Graphite & 6 & 99 \\
\hline
\end{tabular}

${ }^{a}$ Reaction conditions: $1 \mathrm{mmol}$ of acid, $2 \mathrm{mmol}$ of alcohol, $10 \mathrm{wt} \%$ or $0 \mathrm{wt} \%$ catalyst (based on acid), conducted in a sealed tube. ${ }^{b}$ Conversion of acid, determined by ${ }^{1} \mathrm{H}$ NMR; ${ }^{c}$ Selectivity determined by ${ }^{1} \mathrm{H}$ NMR. ${ }^{d}$ Prepared by original Hummers method. ${ }^{e}$ Prepared by Tour method.

\section{2 氧化石墨的结构表征}

由改进 Hummers 法 ${ }^{[10]}$ 制备的 GO 用 IR, XRD, XPS 和元素分析进行结构表征. IR 谱图(图 2)中, $3400(\mathrm{OH})$, $1739(\mathrm{C}=\mathrm{O}), 1227(\mathrm{C}-\mathrm{O}-\mathrm{C})$ 及 $1056(\mathrm{C}-\mathrm{O}) \mathrm{cm}^{-1}$ 等特 征峰的出现证明了石墨的氧化. XRD 谱图(图 3)中在 11.46 处的宽峰为 GO 的特征峰. 元素分析结果显示 $\mathrm{GO}$ 的碳氧元素含量比为 1.11 , 这表明通过强氧化剂的作用 下, GO 结构中含有了大量含氧官能团. XPS 谱图(图 4) 也显示各含氧官能团的存在.

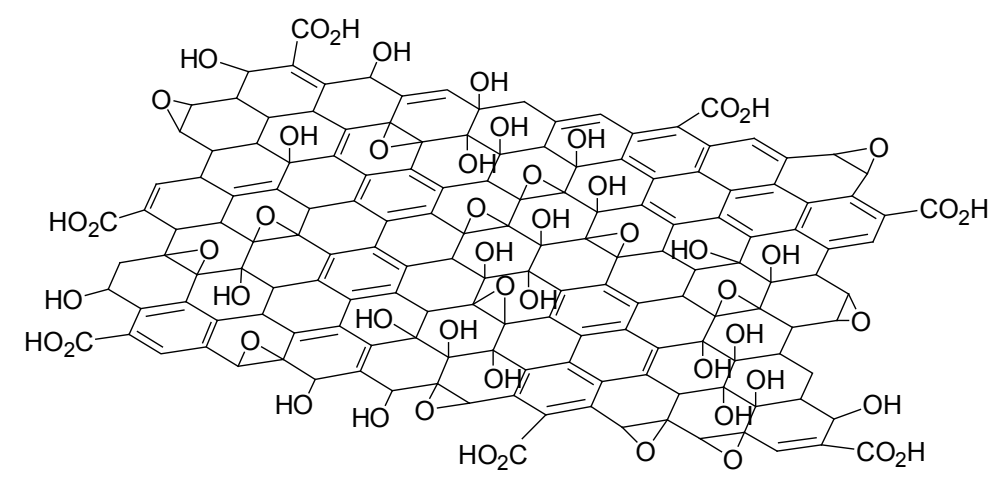

图 1 单层氧化石墨(氧化石墨烯)结构模型

Figure 1 The schematic model of GO monolayer (graphene oxide) 


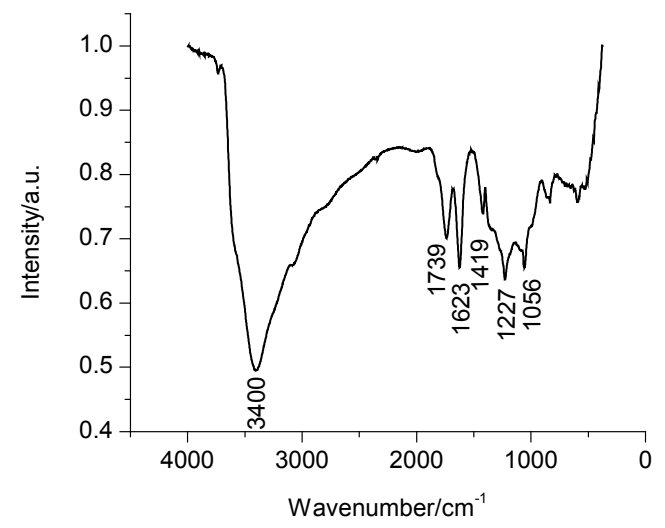

图 $2 \mathrm{GO}$ 的红外谱图

Figure 2 FT-IR spectra of GO

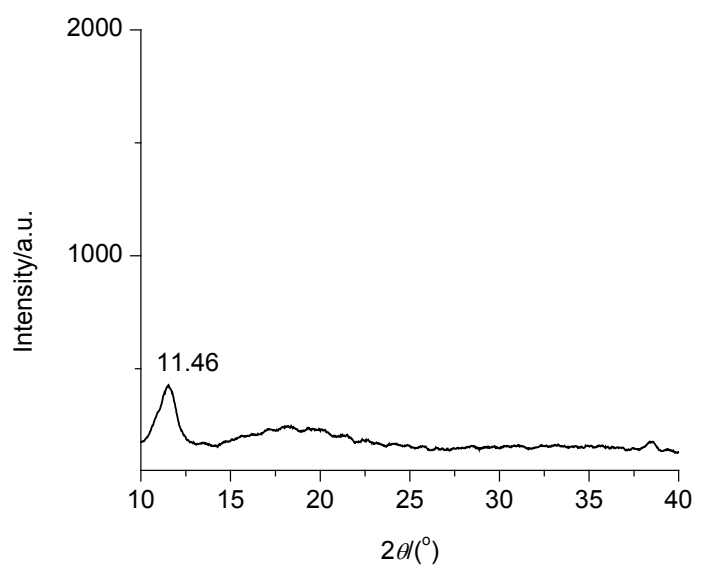

图3 GO的XRD谱图

Figure 3 XRD pattern of GO

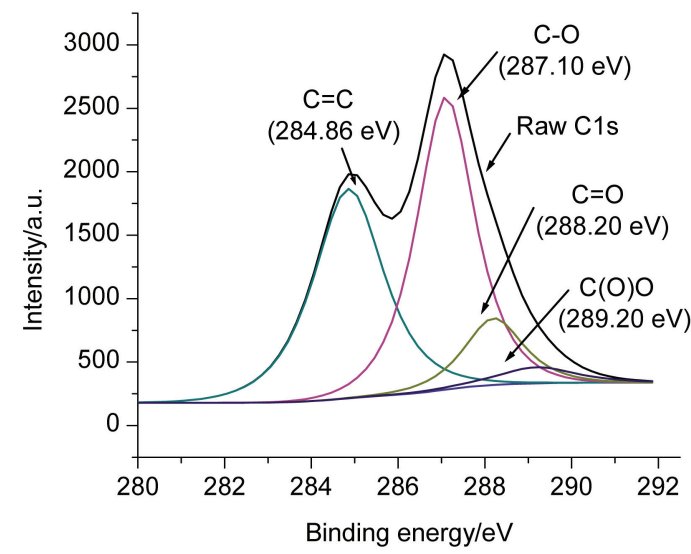

图 $4 \mathrm{GO}$ 的 $\mathrm{C} 1 \mathrm{~s}$ XPS 谱图

Figure 4 The C1s XPS spectrum of graphite oxide

\section{3 实验条件的确定}

我们根据反应底物的特点确定实验条件. 对于大部 分伯醇与羧酸的酯化, 反应在醇的沸点下回流进行. 对 于沸点超过 $100{ }^{\circ} \mathrm{C}$ 的伯醇的酯化, 我们将反应温度选 为 $100{ }^{\circ} \mathrm{C}$, 因为酯化反应生成的水可能与醇和羧酸形
成共沸混合物，而该共沸混合物的沸点通常接近 100 ${ }^{\circ} \mathrm{C}$. 根据我们以往的研究, 升高温度易导致仲醇的脱水 反应，故对于仲醇的酯化，我们将反应温度降低至 80 ${ }^{\circ} \mathrm{C}$. 根据 TLC 跟踪反应进程情况，我们将反应时间统一 为 $9 \mathrm{~h}$.

\section{4 底物扩展情况}

确定实验条件之后, 我们对酯化反应的底物进行扩 展. 实验结果表明, 当羒酸的苯环上连有给电子基团时 (表 2, Entry 2), 产率只有 $67 \%$; 而当羧酸的苯环上连有 $\mathrm{NO}_{2}, \mathrm{Cl}$ 这样的吸电子基团时(表 2, Entries 3 5), 产率 提高至 90\% 94\%, 这可能是因为吸电子基团使炭基碳 原子带有更多的正电性，有利于醇的亲核进攻. 环己烷 甲酸与甲醇以及 2-苯乙醇的酯化(表 2, Entries 6,7)所得 产率分别为 $61 \%$ 和 $93 \%$, 虽然反应中少量环已烷甲酸脱 氢生成苯甲酸, 但酯化反应的选择性很高. 肉桂酸与甲 醇的酯化(表 2, Entry 8)所得产率为 74\%，碳碳双键不发 生反应. 3-苯丙酸、苯氧乙酸、1-芸乙酸与甲醇的酯化(表 2, Entries 9 11)产率达 83\% 90\%. 我们还对链状脂肪 酸的酯化进行了探讨. 乙酸与正辛醇的酯化(表 2, Entry 12)产率为 $80 \%$, 硬脂酸与甲醇和乙醇的酯化(表 2, Entries 13,14$)$ 产率高达 $95 \%$ 和 $98 \%$. 酸值较高的植物油在 制备生物柴油时, 需要经过酯化这一过程, 硬脂酸及一 些不饱和脂肪酸的酯化为其中的典型反应，因此上述反 应结果反映了氧化石墨在生物柴油制备中的应用前景. 由于仲醇(表 2, Entries 16, 17)的脱水, 导致其酯化产率 明显低于伯醇，环己醇和仲辛醇的乙酸酯产率分别为 $62 \%$ 和 $60 \%$. 叔醇难进行酯化反应, 在加入 $4 \AA$ 分子篮 时, 叔丁醇、叔戊醇与 4-硝基苯甲酸的酯化反应产物产 率均低于 10\%(表 2, Entries 18，19). 对照实验表明分子 篮对这一反应无催化作用，仅用作吸水剂. 由于酚羟基 中氧原子的亲核性较低，苯酚不与乙酸反应，4-甲氧基 苯酚的反应也不理想(表 2, Entries 20,21).

\section{5 酯交换反应}

我们将由改进 Hummers 法 ${ }^{[10]}$ 制备的氧化石墨应用 于酯交换反应，结果如表 3 所示. 苯乙醇、正辛醇与甲 酸乙酯和乙酸乙酯反应都以较高收率得到酯交换产物 (表 3, Entries 1 3). 三硬脂酸甘油酯与甲醇在回流条件 下转化率较低, 若在加压管中 $120{ }^{\circ} \mathrm{C}$ 加热 $12 \mathrm{~h}$ (表 3, Entry 4), 产率可达 $74 \%$. 除与甲醇、乙醇等低碳醇反应 外，三硬脂酸甘油酯与正丁醇的反应也是制备生物柴油 的典型反应，我们对其进行了尝试。在正丁醇(表 3, Entry 5)回流条件下，三硬脂酸甘油酯接近全部转化，分 离产率可达 $83 \%$. 综上所述, 氧化石墨可以用作高级脂 肪酸的酯化和高级脂肪酸甘油酯的酯交换反应的高效 
表 2 羧酸与醇和酚的酯化反应 ${ }^{a}$

Table 2 Esterification of carboxylic acids with alcohols and phenols

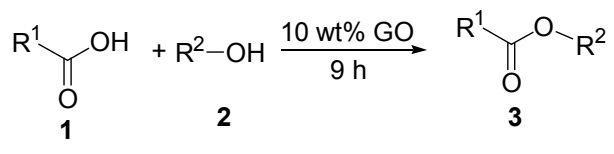

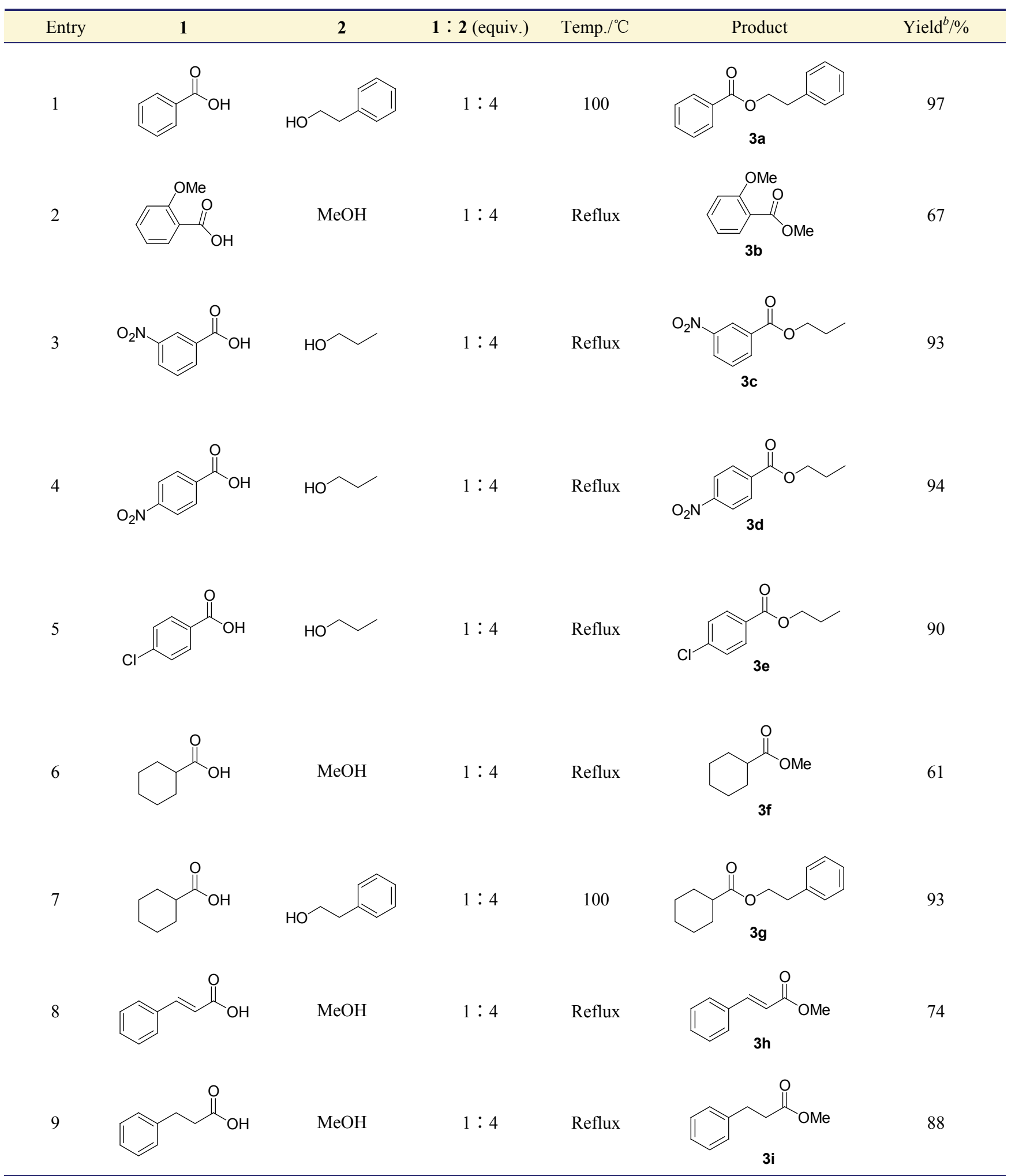




\begin{tabular}{|c|c|c|c|c|c|c|}
\hline Entry & 1 & 2 & $1: 2$ (equiv.) & Temp. $/{ }^{\circ} \mathrm{C}$ & Product & Yield $^{b} / \%$ \\
\hline 10 & & $\mathrm{MeOH}$ & $1: 4$ & Reflux & 3j & 83 \\
\hline 11 & & $\mathrm{MeOH}$ & $1: 4$ & Reflux & $3 \mathbf{k}$ & 90 \\
\hline 12 & & $\mathrm{HO}^{+} \mathrm{H}_{7}$ & $4: 1$ & 80 & 31 & 80 \\
\hline 13 & & $\mathrm{MeOH}$ & $1: 4$ & Reflux & $3 m$ & 95 \\
\hline 14 & & $\mathrm{EtOH}$ & $1: 4$ & Reflux & $3 n$ & 98 \\
\hline 15 & & $\mathrm{MeOH}$ & $1: 8$ & Reflux & 30 & 73 \\
\hline 16 & & & $3: 1$ & 80 & $3 p$ & $62^{c}$ \\
\hline 17 & & & $3: 1$ & 80 & $3 q$ & $60^{c}$ \\
\hline 18 & & $t-\mathrm{BuOH}$ & - & Reflux & $3 r$ & $<5^{d}$ \\
\hline 19 & & & - & Reflux & $3 s$ & $8^{d}$ \\
\hline
\end{tabular}




\begin{tabular}{|c|c|c|c|c|c|c|}
\hline Entry & 1 & 2 & $1: 2$ (equiv.) & Temp $/{ }^{\circ} \mathrm{C}$ & Product & Yield $^{b} / \%$ \\
\hline 20 & & & - & Reflux & & $\mathrm{NR}^{e}$ \\
\hline 21 & & & - & Reflux & & $7^{e}$ \\
\hline
\end{tabular}

$\overline{{ }^{a}}$ The amount of GO is $10 \mathrm{wt} \%$ of the limiting reactant. ${ }^{b}$ Isolated yield. ${ }^{c}$ Reaction time: $12 \mathrm{~h}^{d} 3 \mathrm{mmol}$ of $\mathbf{1}, 1 \mathrm{~mL}$ of $2,0.1 \mathrm{~g} \mathrm{of} 4 \AA \mathrm{MS}, 12 \mathrm{~h}{ }^{e} 1 \mathrm{~mL}$ of $\mathbf{1}, 3 \mathrm{mmol}$ of $2,12 \mathrm{~h}$.

催化剂, 在生物柴油生产领域, 尤其对由酸值较高的植的酯交换产物生成, 表明氧化石墨具有确定的催化作 物油制备生物柴油这一过程有着很大的潜在应用价值．用. 在空白实验中(表 3, Entry 6), 没有乙酸乙酯与 2-苯乙醇

表 3 酯与醇的酯交换反应

Table 3 Transesterification of esters with alcohols

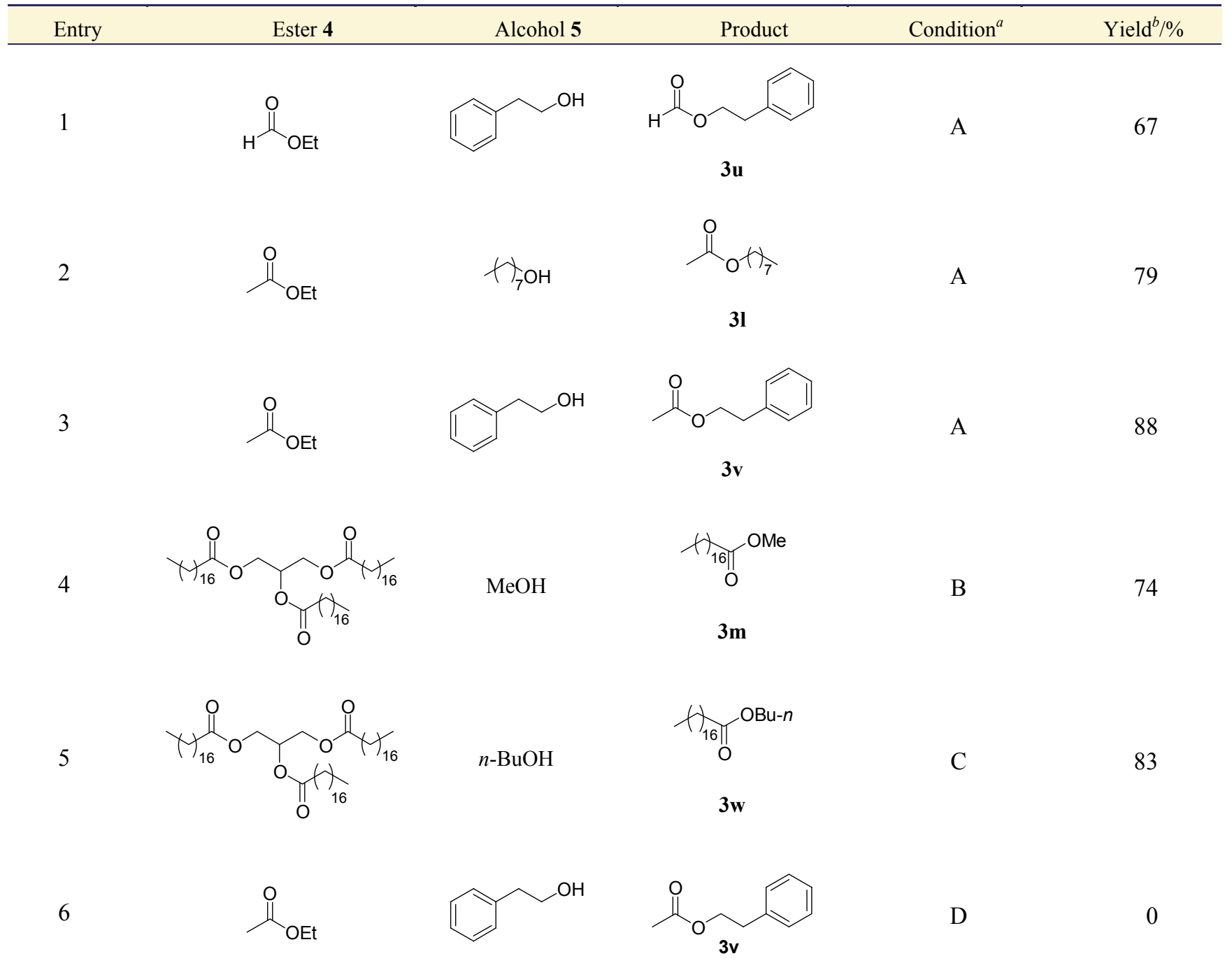

${ }^{a}$ Condition A: $30 \mathrm{mmol}$ of 4, $3 \mathrm{mmol}$ of 5, GO (10 wt\% of 5), reflux, $9 \mathrm{~h}$; Condition B: $0.3 \mathrm{mmol}$ of 4, 9 mmol of 5, GO (10 wt\% of 4), $120{ }^{\circ} \mathrm{C}, 12 \mathrm{~h}$, performed in a pressure tube; Condition C: $0.3 \mathrm{mmol}$ of $\mathbf{4}, 9 \mathrm{mmol}$ of $\mathbf{5}$, GO (10 wt $\%$ of 4$)$, reflux, $12 \mathrm{~h}$; Condition D: without GO under condition A. ${ }^{b}$ Isolated yield. 


\section{2 结论}

改进 Hummers 法制备的氧化石墨用作伯醇、仲醇 的酯化反应催化剂, 催化剂用量少, 催化效率高, 能与 多种官能团兼容, 反应操作简便, 产物提纯简单或可省 略, 通常具有很高选择性和收率. 其在酯交换反应中也 表现出较高的催化活性, 但对叔醇、酚的酯化反应催化 效果欠佳. 氧化石墨作为一种良好的固体弱酸性催化 剂, 具有容易制备、廉价、环境友好、底物适用范围广 的优点. 此催化反应在生物柴油的生产领域也具有潜在 应用价值.

\section{3 实验部分}

\section{1 仪器与试剂}

瑞士 Bruker 公司 AVANCE III (400 MHz)核磁共振 仪(TMS 作内标, $\mathrm{CDCl}_{3}$ 作溶剂), 德国 Bruker 公司 ALPHA 型红外光谱仪 ( $\mathrm{KBr}$ 压片), 北京大学仪器厂 BDX-3300 型 X 射线衍射仪, 德国 Elementar 公司 Vario Micro cube 型元素分析仪, 日本 PHI 公司 5000 VersaProbe 型 $X$ 射线光电子能谱仪.

石墨粉为市售高纯试剂(含量 $\geqslant 99.99 \%) ; \mathrm{KMnO}_{4}$, $\mathrm{HCl}, \mathrm{H}_{2} \mathrm{SO}_{4}, \mathrm{NaNO}_{3}, 30 \% \mathrm{H}_{2} \mathrm{O}_{2}$ 为市售优级纯试剂; 其它 试剂为市售分析纯. 柱层析使用 200 300 目硅胶.

\section{2 实验方法}

\section{2 .1 氧化石墨的制备}

根据改进 Hummers 法 ${ }^{[10]}$ 制备氧化石墨. 冰浴条件 下, 将浓 $\mathrm{H}_{2} \mathrm{SO}_{4}(69 \mathrm{~mL})$ 缓慢加至石墨 $(3.0 \mathrm{~g})$ 与 $\mathrm{NaNO}_{3}$ $(1.5 \mathrm{~g})$ 的混合体系中. 机械摚拌下, 向反应体系中分批 加入 $\mathrm{KMnO}_{4}(9.0 \mathrm{~g})$, 防止反应体系温度超过 $20{ }^{\circ} \mathrm{C}, 35$ ${ }^{\circ} \mathrm{C}$ 反应 $7 \mathrm{~h}$ 后, 使反应体系降至室温并再次缓慢加入 $\mathrm{KMnO}_{4}(9.0 \mathrm{~g}), 35{ }^{\circ} \mathrm{C}$ 反应 $12 \mathrm{~h}$. 反应完成后, 降至室温, 将反应体系倒入 $420 \mathrm{~mL}$ 去离子水中, 缓慢滴加 $3 \mathrm{~mL}$ $30 \% \mathrm{H}_{2} \mathrm{O}_{2}$, 体系变为亮黄色并有气泡产生. 抽滤, 依次 反复用 $200 \mathrm{~mL} 30 \% \mathrm{HCl}, 200 \mathrm{~mL}$ 去离子水洗涤固体, 直 至滤液 $\mathrm{pH}$ 为 6 7. 弃去滤液, 将固体置于真空干燥器 中干燥至恒重. 所得氧化石墨为深棕色粉末状固体.

\subsection{2 酯化反应实验方法}

伯醇的酯化通法. 向 $25 \mathrm{~mL}$ 圆底烧瓶中加入 3 $\mathrm{mmol} \mathrm{1,12} \mathrm{mmol}$ 或 $24 \mathrm{mmol} 2, \mathrm{GO}$ (1 质量的 $10 \%)$, 反 应在回流或设定温度下摚拌 $9 \mathrm{~h}$. 反应结束后, 过滤, 用 $\mathrm{CH}_{2} \mathrm{Cl}_{2}(10 \mathrm{~mL} \times 2)$ 洗涤 $\mathrm{GO}$, 合并滤液, 旋蒸除去有机 溶剂得产物 3a $~ 3 \mathrm{k}, 3 \mathrm{~m}$ 和 $3 \mathrm{n}$. 必要时以硅胶柱层析提 纯产物. 31 和 30 的后处理同仲醇.

仲醇的酯化通法. 向 $25 \mathrm{~mL}$ 圆底烧瓶中加入 3 mmol 2, 9 mmol 1, GO (2 质量的 $10 \%), 80{ }^{\circ} \mathrm{C}$ 下摚拌 12 h. 反应结束后过滤, 用 $\mathrm{CH}_{2} \mathrm{Cl}_{2}(10 \mathrm{~mL} \times 2)$ 洗涤 $\mathrm{GO}$, 合 并滤液, 用饱和 $\mathrm{NaHCO}_{3}(6 \mathrm{~mL} \times 2)$ 洗涤, 分液, 有机相 用无水 $\mathrm{NaSO}_{4}$ 干燥. 旋蒸除去溶剂得纯产品 $\mathbf{3 p}$ 和 $\mathbf{3 q}$.

叔醇的酯化通法. 向 $25 \mathrm{~mL}$ 圆底烧瓶中加入 3

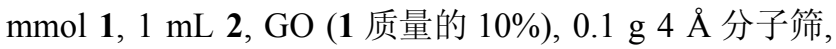
搅拌回流 $12 \mathrm{~h}$. 后处理同伯醇, 得纯产品 $3 \mathbf{r}$ 和 $3 \mathbf{s}$.

酚的酯化通法. 向 $25 \mathrm{~mL}$ 圆底烧瓶中加入 $1 \mathrm{~mL} \mathrm{1,3}$ $\mathrm{mmol} 2, \mathrm{GO}(2$ 质量的 $10 \%)$, 摚拌回流 $12 \mathrm{~h}$. 后处理同仲 醇, 经硅胶柱层析得纯产品 $\mathbf{3 t}$.

\subsection{3 酯交换反应的实验方法}

对于 $3 \mathbf{u}, 3 \mathbf{3 l}, 3 \mathbf{v}$, 向 $50 \mathrm{~mL}$ 的圆底烧瓶中加入 $3 \mathrm{mmol}$ $5,30 \mathrm{mmol}$ 4, GO (5 质量的 $10 \%)$, 搅拌回流 $9 \mathrm{~h}$. 反应结 束后, 过滤, 用 $\mathrm{CH}_{2} \mathrm{Cl}_{2}(10 \mathrm{~mL} \times 2)$ 洗涤 $\mathrm{GO}$, 收集滤液, 旋蒸除去溶剂得纯产品. $3 \mathrm{u}$ 需要用硅胶柱层析提纯.

对于 $3 \mathrm{~m}$ 和 $3 \mathrm{w}$, 向 $50 \mathrm{~mL}$ 的圆底烧瓶 $(3 \mathrm{~m}$ 需在加 压管中合成)中加入 $0.3 \mathrm{mmol} \mathrm{4}, 9 \mathrm{mmol} \mathrm{5,GO}$ (4 质量的 $10 \%), 120{ }^{\circ} \mathrm{C}$ 加热摚拌 $12 \mathrm{~h}$. 反应结束后, 用乙醚 $(10$ $\mathrm{mL} \times 2)$ 洗涤 $\mathrm{GO}$, 合并滤液, 用饱和食盐水 $(6 \mathrm{~mL} \times 2)$ 洗 涤, 有机相用无水 $\mathrm{NaSO}_{4}$ 干燥, 旋蒸除去溶剂得纯产 品.

所有产物都为已知化合物, 且通过 ${ }^{1} \mathrm{H}$ NMR 和 ${ }^{13} \mathrm{C}$ NMR 表征, 核磁数据均与文献报道数据相符. 其中部 分产物的核磁数据如下.

环已烷甲酸-2-苯乙酯(3g) $)^{[17 \mathrm{7a}]}:{ }^{1} \mathrm{H} \mathrm{NMR}\left(\mathrm{CDCl}_{3}, 400\right.$ MHz) $\delta: 1.15 \sim 1.30(\mathrm{~m}, 3 \mathrm{H}), 1.36 \sim 1.45(\mathrm{~m}, 2 \mathrm{H}), 1.59 \sim$ $1.62(\mathrm{~m}, 1 \mathrm{H}), 1.72(\mathrm{~d}, J=10.6 \mathrm{~Hz}, 2 \mathrm{H}), 1.85$ (d, $J=12.6$ $\mathrm{Hz}, 2 \mathrm{H}), 2.26$ (tt, $J=3.5,11.2 \mathrm{~Hz}, 1 \mathrm{H}), 2.92(\mathrm{t}, J=7.0 \mathrm{~Hz}$, 2H), 4.27 (t, $J=7.0 \mathrm{~Hz}, 2 \mathrm{H}), 7.19 \sim 7.22(\mathrm{~m}, 3 \mathrm{H}), 7.27 \sim$ $7.30(\mathrm{~m}, 2 \mathrm{H}) ;{ }^{13} \mathrm{C} \mathrm{NMR}\left(\mathrm{CDCl}_{3}, 100 \mathrm{MHz}\right) \delta: 25.29(2 \mathrm{C})$, $25.67,28.85$ (2C), 35.06, 42.96, 64.38, 126.33, 128.28 (2C), 128.75 (2C), 137.81, 175.57.

肉桂酸甲酯(3h) $)^{[17 b]}:{ }^{1} \mathrm{H} \mathrm{NMR}\left(\mathrm{CDCl}_{3}, 400 \mathrm{MHz}\right) \delta$ : $3.81(\mathrm{~s}, 3 \mathrm{H}), 6.44(\mathrm{~d}, J=16.0 \mathrm{~Hz}, 1 \mathrm{H}), 7.37 \sim 7.39$ (m, $3 \mathrm{H}), 7.51 \sim 7.53(\mathrm{~m}, 2 \mathrm{H}), 7.70(\mathrm{~d}, J=16.0 \mathrm{~Hz}, 1 \mathrm{H}) ;{ }^{13} \mathrm{C}$ NMR $\left(\mathrm{CDCl}_{3}, 100 \mathrm{MHz}\right) \delta: 51.75,117.89,128.15$ (2C), 128.96 (2C), 130.37, 134.47, 144.94, 167.48.

苯氧乙酸甲酯 $(\mathbf{3 j})^{[17 \mathrm{c}]}$ : ${ }^{1} \mathrm{H} \mathrm{NMR}\left(\mathrm{CDCl}_{3}, 400 \mathrm{MHz}\right)$ $\delta: 3.74(\mathrm{~s}, 3 \mathrm{H}), 4.58$ (s, 2H), 6.88 (d, $J=7.9 \mathrm{~Hz}, 2 \mathrm{H}), 6.96$ (t, $J=7.4 \mathrm{~Hz}, 1 \mathrm{H}), 7.26(\mathrm{t}, J=8.0 \mathrm{~Hz}, 2 \mathrm{H}) ;{ }^{13} \mathrm{C}$ NMR $\left(\mathrm{CDCl}_{3}, 100 \mathrm{MHz}\right) \delta: 51.98,65.10,114.52$ (2C), 121.62, 129.46 (2C), 157.72, 169.27.

1-菜乙酸甲酯 $(\mathbf{3 k})^{[17 b]}$ : ${ }^{1} \mathrm{H} \mathrm{NMR}\left(\mathrm{CDCl}_{3}, 400 \mathrm{MHz}\right)$ $\delta: 3.73(\mathrm{~s}, 3 \mathrm{H}), 4.14(\mathrm{~s}, 2 \mathrm{H}), 7.47 \sim 7.63(\mathrm{~m}, 4 \mathrm{H}), 7.86(\mathrm{~d}$, $J=7.6 \mathrm{~Hz}, 1 \mathrm{H}), 7.93$ (d, $J=8.0 \mathrm{~Hz}, 1 \mathrm{H}), 8.09$ (d, $J=8.3$ $\mathrm{Hz}, 1 \mathrm{H}) ;{ }^{13} \mathrm{C} \mathrm{NMR}\left(\mathrm{CDCl}_{3}, 100 \mathrm{MHz}\right) \delta: 38.90,51.96$, 
$123.72,125.42,125.72,126.33,127.94,128.02,128.68$, $130.50,132.07,133.79,171.88$.

乙酸仲辛酯 $(\mathbf{3 q})^{[18 \mathrm{a}]}:{ }^{1} \mathrm{H} \mathrm{NMR}\left(\mathrm{CDCl}_{3}, 400 \mathrm{MHz}\right) \delta$ : $0.87(\mathrm{t}, J=6.5 \mathrm{~Hz}, 3 \mathrm{H}), 1.19(\mathrm{~d}, J=6.3 \mathrm{~Hz}, 3 \mathrm{H}), 1.26 \sim$ $1.32(\mathrm{~m}, 8 \mathrm{H}), 1.41 \sim 1.48(\mathrm{~m}, 1 \mathrm{H}), 1.53 \sim 1.58(\mathrm{~m}, 1 \mathrm{H})$, $2.01(\mathrm{~s}, 3 \mathrm{H}), 4.83 \sim 4.91(\mathrm{~m}, 1 \mathrm{H}) ;{ }^{13} \mathrm{C} \mathrm{NMR}\left(\mathrm{CDCl}_{3}, 100\right.$ MHz) $\delta$ : 14.17, 20.08, 21.50, 22.70, 25.49, 29.25, 31.87, $36.06,71.20,170.91$.

4-硝基苯甲酸叔戊酯(3s $)^{[18 b]}$ : ${ }^{1} \mathrm{H}$ NMR $\left(\mathrm{CDCl}_{3}, 400\right.$ MHz) $\delta: 0.98(\mathrm{t}, J=7.5 \mathrm{~Hz}, 3 \mathrm{H}), 1.59(\mathrm{~s}, 6 \mathrm{H}), 1.94$ (q, $J=$ $7.4 \mathrm{~Hz}, 2 \mathrm{H}), 8.15$ (d, $J=8.8 \mathrm{~Hz}, 2 \mathrm{H}), 8.26$ (d, $J=8.9 \mathrm{~Hz}$, $2 \mathrm{H}) ;{ }^{13} \mathrm{C} \mathrm{NMR}\left(\mathrm{CDCl}_{3}, 100 \mathrm{MHz}\right) \delta: 8.47,25.68,33.75$, $85.26,123.54,130.61,137.57,150.40,163.79$.

乙酸-4-甲氧基苯酚酯(3t) ${ }^{[18 \mathrm{c}]}:{ }^{1} \mathrm{H} \mathrm{NMR}\left(\mathrm{CDCl}_{3}, 400\right.$ $\mathrm{MHz}) \delta: 2.28(\mathrm{~s}, 3 \mathrm{H}), 3.80(\mathrm{~s}, 3 \mathrm{H}), 6.89(\mathrm{~d}, J=9.1 \mathrm{~Hz}$, 2H), 7.00 (d, $J=9.0 \mathrm{~Hz}, 2 \mathrm{H}) ;{ }^{13} \mathrm{C} \mathrm{NMR}\left(\mathrm{CDCl}_{3}, 100 \mathrm{MHz}\right)$ $\delta: 21.20,55.70,114.58,122.44,144.29,157.37,170.06$.

甲酸-2-苯乙酯 $(3 \mathbf{u})^{[18 \mathrm{~d}]}:{ }^{1} \mathrm{H} \mathrm{NMR}\left(\mathrm{CDCl}_{3}, 400 \mathrm{MHz}\right)$ $\delta: 2.93(\mathrm{t}, J=7.0 \mathrm{~Hz}, 2 \mathrm{H}), 4.35(\mathrm{t}, J=7.0 \mathrm{~Hz}, 2 \mathrm{H}), 7.19 \sim$ $7.30(\mathrm{~m}, 5 \mathrm{H}), 7.97(\mathrm{~s}, 1 \mathrm{H}) ;{ }^{13} \mathrm{C} \mathrm{NMR}\left(\mathrm{CDCl}_{3}, 100 \mathrm{MHz}\right) \delta$ : 34.86, 64.23, 126.65, 128.51 (2C), 128.82 (2C), 137.42, 160.84 .

硬脂酸丁酯 $(\mathbf{3 w})^{[18 \mathrm{e}]}$ : ${ }^{1} \mathrm{H} \mathrm{NMR}\left(\mathrm{CDCl}_{3}, 400 \mathrm{MHz}\right) \delta$ : $0.87(\mathrm{t}, J=6.7 \mathrm{~Hz}, 3 \mathrm{H}), 0.92(\mathrm{t}, J=7.4 \mathrm{~Hz}, 3 \mathrm{H}), 1.24 \sim$ $1.27(\mathrm{~m}, 28 \mathrm{H}), 1.32 \sim 1.42(\mathrm{~m}, 2 \mathrm{H}), 1.56 \sim 1.63(\mathrm{~m}, 4 \mathrm{H})$, $2.27(\mathrm{t}, J=7.5 \mathrm{~Hz}, 2 \mathrm{H}), 4.06(\mathrm{t}, J=6.7 \mathrm{~Hz}, 2 \mathrm{H}) ;{ }^{13} \mathrm{C} \mathrm{NMR}$ $\left(\mathrm{CDCl}_{3}, 100 \mathrm{MHz}\right) \delta: 13.62,14.02,19.17,22.70,25.02$, $29.19,29.31,29.41,29.51,29.64,29.74,30.79,31.97$, $34.30,63.91,173.60$.

\section{References}

[1] Otera, J.; Nishikido, J. In Esterification: Methods, Reactions, and Applications, 2nd ed., Wiley-VCH, Weinheim, Japan, 2010, pp. $293 \sim 320$.

[2] Borges, M. E.; Diaz, L. Renewable Sustainable Energy Rev. 2012, 16, 2839.

[3] (a) Smith, K.; El-Hiti, G. A.; Jayne, A. J.; Butters, M. Org. Biomol. Chem. 2003, $1,1560$.

(b) Melero, J. A.; Iglesias, J.; Morales, G. Green Chem. 2009, 11, 1285.

[4] Okuhara, T. Chem. Rev. 2002, 102, 3641.

[5] (a) Guo, Z.; Lue, B.; Thomasen, K.; Meye, A. S.; Xu, X. Green Chem. 2007, 9, 1362. (b) Li, J.; Wang, J.; Zhang, L.-X.; Gu, S.-S.; Wu, F.-A.; Guo, Y.-W. Chin. J. Org. Chem. 2012, 32, 1186 (in Chinese)

(李晶, 王俊, 张否霞, 顾双双, 吴福安, 郭跃伟, 有机化学, 2012, 32, 1186.)

[6] (a) Salinier, V.; Niccolai, G. P.; Dufaud, V.; Basset, J.-M. Adv. Synth. Catal. 2009, 351, 2168.

(b) Zeng, T.; Song, G.; Li, C.-J. Chem. Commun. 2009, 6249.

[7] (a) Toda, M.; Takagaki, A.; Okamura, M.; Kondo, J. N.; Hayashi, S.; Domen, K.; Hara, M. Nature 2005, 438, 178.

(b) Liu, F.; Sun, J.; Zhu, L.; Meng, X.; Qi, C., Xiao, F.-S. J. Mater. Chem. 2012, 22, 5495.

(c) Nevskaia, D. M.; Martín-Aranda, R. M. Catal. Lett. 2003, 87, 143.

[8] (a) Stankovich, S.; Dikin, D. A.; Dommett, G. H. B.; Kohlhaas, K. M.; Zimney, E. J.; Stach, E. A.; Piner, R. D.; Nguyen, S. T.; Ruoff, R. S. Nature 2006, 442, 282.

(b) Wei, Z.; Wang, D.; Kim, S.; Kim, S.-Y.; Hu, Y.; Yakes, M. K.; Laracuente, A. R.; Dai, Z.; Marder, S. R.; Berger, C.; King, W. P.; Heer, W. A.; Sheehan, P. E.; Riedo, E. Science 2010, 328, 1373.

[9] Hummers, W. S.; Offeman, R. E. J. Am. Chem. Soc. 1958, 80, 1339.

[10] Marcano, D. C.; Kosynkin, D. V.; Berlin, J. M.; Sinitskii, A.; Sun, Z. Z.; Slesarev, A.; Alemany, L. B.; Lu, W.; Tour, J. M. ACS Noano 2010, 4, 4806.

[11] Dreyer, D. R.; Park, S.; Bielawski, C. W.; Ruoff, R. S. Chem. Soc Rev. 2010, 39, 228

[12] Dreyer, D. R.; Jia, H. P., Bielawski, C. W. Angew. Chem., Int. Ed. 2010, 49, 6813.

[13] Huang, H.; Huang, J.; Liu, Y. M.; He, H. Y.; Cao, Y.; Fan, K. N. Green Chem. 2012, 14, 930.

[14] (a) Kumar, A. V.; Rao, K. R. Tetrahedron Lett. 2011, 52, 5188.

(b) Verma, S.; Mungse, H. P.; Kumar, N.; Choudhary, S.; Jain, S. L.; Sain, B.; Khatri, O. P. Chem. Commun. 2011, 47, 12673.

(c) Dhakshinamoorthy, A.; Alvaro, M.; Concepción, P.; Fornés, V.; Garcia, H. Chem. Commun. 2012, 48, 5443.

[15] Zhang, X.; Xu, L.; Wang, X.-T.; Ma, N.; Sun, F.-F. Chin. J. Chem. 2012, 30, 1525.

[16] Zu, Y. H.; Tang, J. Y.; Zhu, W. C.; Zhang, M.; Liu, G.; Liu, Y.; Zhang, W. X.; Jia, M. J. Bioresour. Technol. 2011, 102, 8939.

[17] (a) Tian, J.; Gao, W. C.; Zhou, D. M.; Zhang, C. Org. Lett. 2012, 14, 3020.

(b) Li, L. C.; Ren, J.; Liao, T. G. Jiang, J. X.; Zhu, H. J. Eur. J. Org. Chem. 2007, 1026.

(c) Li, J. T.; Li, H. Y.; Li, H. Z. J. Chem. Res. 2004, 6, 416.

[18] (a) Ratnam, K. J.; Reddy, R. S.; Sekhar, N. S.; Kantam, M. L.; Figueras, F. J. Mol. Catal. A: Chem. 2007, 276, 230

(b) Brown, H. C.; Rao, C. G.; Ravindranathanl, M. J. Org. Chem. 1978, 43, 4939.

(c) Qiu, D.; Zheng, Z. T.; Mo, F. Y.; Xiao, Q.; Tian, Y.; Zhang, Y.; Wang, J. B. Org. Lett. 2011, 13, 4988.

(d) Wang, J. Q.; He, L. N.; Miao, C. X. Green Chem. 2009, 11, 1013.

(e) Iwasaki, T.; Maegawa, Y.; Hayashi, Y.; Ohshima, T.; Mashima, K. J. Org. Chem. 2008, 73, 5147.

(Qin, X.) 